\title{
Venous Thromboembolism and Mild Thrombocytopenia after ChAdOx1 nCoV-19 Vaccination
}

\author{
Nadia Gabarin ${ }^{1, *}$ Sarah Patterson ${ }^{2, *}$ Menaka Pai ${ }^{1,2,3,4}$ Tamoor Afzaal ${ }^{1}$ Ishac Nazy ${ }^{1,3}$ \\ Jo-Ann I. Sheppard ${ }^{4}$ Donald M. Arnold ${ }^{1,2,3}$ Theodore E. Warkentin ${ }^{1,2,3,4}$
}

${ }^{1}$ Department of Medicine, Michael G. DeGroote School of Medicine,

McMaster University, Hamilton, Ontario, Canada

2 Department of Medicine, Hamilton Health Sciences, Hamilton, Ontario, Canada

${ }^{3}$ McMaster Center for Transfusion Research, McMaster University, Hamilton, Ontario, Canada

${ }^{4}$ Department of Pathology and Molecular Medicine, Michael G. DeGroote School of Medicine, McMaster University, Hamilton, Ontario, Canada

Thromb Haemost 2021;121:1677-1680.

Thrombocytopenia and thrombosis have been reported following vaccination with the ChAdOx1 nCoV-19 (AstraZeneca) vaccine, an adenoviral vector COVID-19 (coronavirus disease 2019) vaccine. ${ }^{1-5}$ Previous cases of vaccine-induced immune thrombotic thrombocytopenia (VITT) have been reported with symptom onset between 5 to 30 days postvaccination, typically with severe thrombocytopenia, unusual thromboses, high mortality, and platelet-activating antibodies against platelet factor 4 (PF4) despite no previous exposure to heparin. ${ }^{1-3}$ Here we report two patients with VITT presenting as lower limb venous thrombosis (one with concurrent pulmonary embolism) and mild thrombocytopenia. One had a late presentation 31 days postvaccination. Both patients were shown to have platelet-activating antibodies in a PF4-dependent serotonin-release assay (SRA).

\section{Case 1}

A 68-year-old male received his first dose of ChAdOx1 nCoV-19 vaccine (day 0 ). On day 31 , he noted left leg swelling and erythema. Two days later he presented to an urgent care center with clinical evidence of left lower limb deep-vein thrombosis (DVT). The D-dimer was $>20 \mathrm{mg} / \mathrm{L}$ (reference

\footnotetext{
* These authors contributed equally to this work.
}

received

June 10, 2021

accepted after revision

August 6, 2021

published online

August 12, 2021
Address for correspondence Theodore E. Warkentin, MD, Room 1-270B, Hamilton Regional Laboratory Medicine Program, Hamilton Health Sciences, Hamilton General Hospital, 237 Barton St. East, Hamilton, Ontario L8L 2X2, Canada (e-mail: twarken@mcmaster.ca).

range: $<0.5 \mathrm{mg} / \mathrm{L}$ ); the platelet count was $116 \times 10^{9} / \mathrm{L}$ (reference range: $150-400 \times 10^{9} / \mathrm{L}$ ); the patient's usual platelet count was $200 \times 10^{9} / \mathrm{L}$ ( -Fig. 1A). He was started on apixaban $10 \mathrm{mg}$ twice-daily as an outpatient; the next day, Doppler ultrasound demonstrated extensive left lower limb DVT involving the mid-superficial femoral vein and extending into the popliteal vein and its trifurcation. He continued apixaban at home, with no bleeding. At follow-up outpatient assessment (day 37), his left leg swelling was reduced; the D-dimer had decreased to $6.1 \mathrm{mg} / \mathrm{L}$. At this time, the polyspecific PF4/polyanion enzyme immunoassay (EIA; Immucor, Dartmouth, Canada) was positive (1.020 optical density [OD] units; reference range: $<0.400)$, as were two immunoglobulin G (IgG)-specific EIAs (PF4/heparin [PF4/H] ${ }^{6}$ and PF4 alone [see the figure legend for the method]), with $>50 \%$ inhibition with high heparin $(100 \mathrm{IU} / \mathrm{mL})$; patient serum induced serotonin release in the presence of PF4 (-Fig. 1A, inset). On day 39, leg swelling had further improved; the D-dimer was $5.6 \mathrm{mg} / \mathrm{L}$ and the platelet count was $160 \times 10^{9} / \mathrm{L}$. Given the persistent relative thrombocytopenia and positive VITT serology, he received $85 \mathrm{~g}$ of intravenous immune globulin (IVIG) $(1 \mathrm{~g} / \mathrm{kg}$ dose [weight: $85 \mathrm{~kg}$; height: $191 \mathrm{~cm}]$ ), with a second dose 24 hours later. His platelet count rose (peak: $234 \times 10^{9} / \mathrm{L}$ ) and the $\mathrm{D}$-dimer progressively decreased. At last assessment on day 147, he remained well,

\footnotetext{
(C) 2021. The Author(s).

This is an open access article published by Thieme under the terms of the Creative Commons Attribution-NonDerivative-NonCommercial-License, permitting copying and reproduction so long as the original work is given appropriate credit. Contents may not be used for commercial purposes, or adapted, remixed, transformed or built upon. (https://creativecommons.org/ licenses/by-nc-nd/4.0/) Georg Thieme Verlag KG, Rüdigerstraße 14, 70469 Stuttgart, Germany
} 

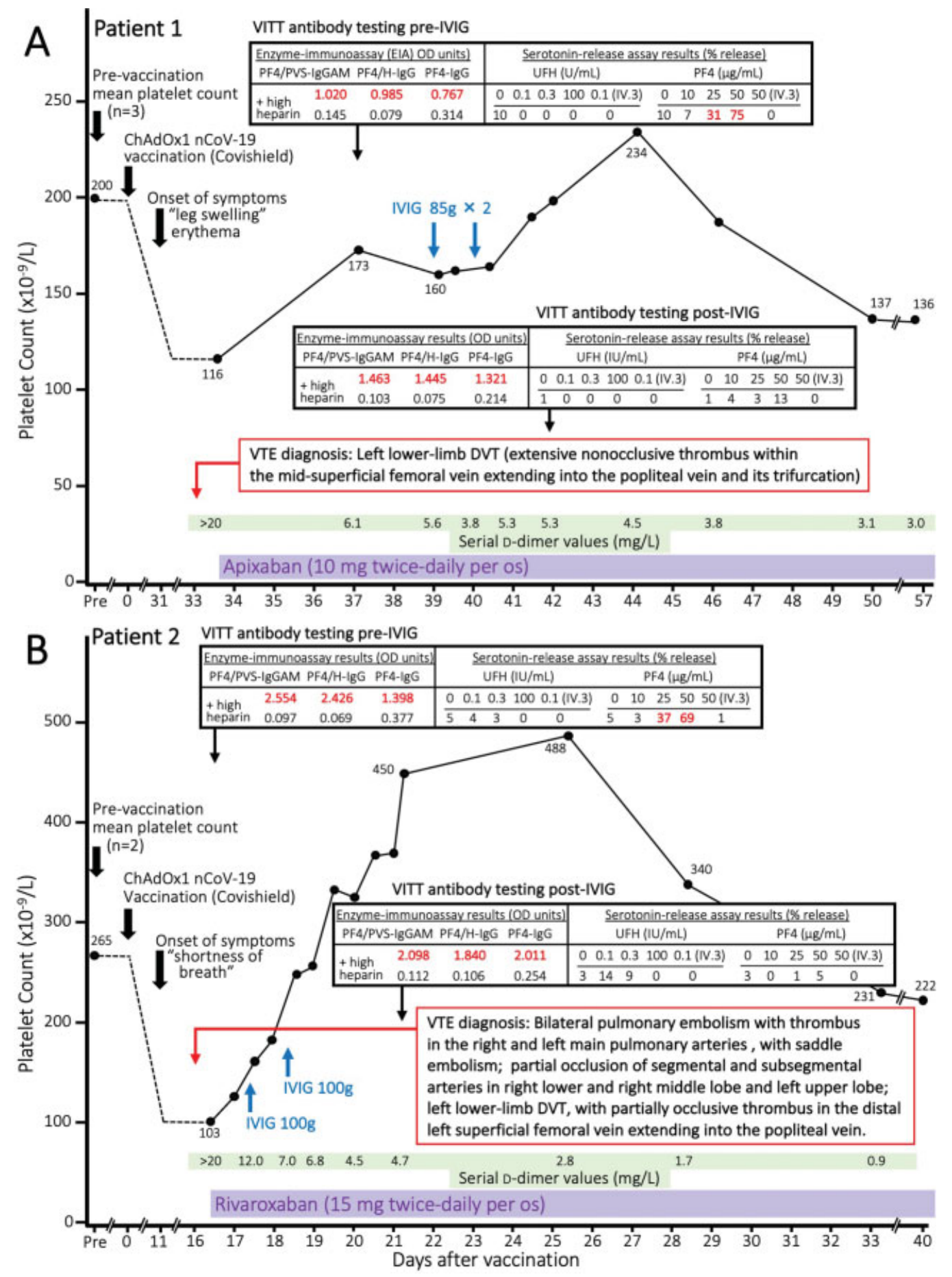

Fig. 1 Clinical course of two patients with vaccine-induced immune thrombotic thrombocytopenia (VITT) with venous thromboembolism and mild thrombocytopenia. For both patients, the baseline ("pre") platelet count represents the mean value of three (patient 1) or two (patient 2) previous platelet count values. "Day 0" indicates the date of vaccination. The inset shows the detailed results of testing for VITT antibodies. Three EIAs are shown: polyspecific (IgGAM) EIA with PF4/polyvinyl sulfonate as the target antigen, as well as two "in-house" IgG-specific EIAs with $\mathrm{PF} 4 /$ heparin $(\mathrm{PF} 4 / \mathrm{H})$ and PF4 alone as target antigens. The IgG-specific ElA used to detect antibodies specific to PF4 alone was modified from a published method, ${ }^{6}$ whereby PF4 alone $(60 \mathrm{\mu g} / \mathrm{mL})$, rather than PF4/heparin complexes, was coated onto the plates, prior to addition of patient sample. +High heparin indicates EIA performed with $100 \mathrm{IU} / \mathrm{mL}$ heparin. For both patients, EIA reactivity remained positive post-IVIG, whereas the platelet activation assays became negative. (A) Patient 1: treatment with high-dose intravenous IVIG was associated with a transient increase in the platelet count (peak, 5 days post-initiation). The patient had no recurrence of thrombosis (last follow-up, day 147). (B) Patient 2: treatment with high-dose IVIG was associated with a transient increase in the platelet count (peak platelet count, 9 days after initiating IVIG). The patient had no recurrence of thrombosis (last follow-up, day 138). IV.3 is the name of the Fc receptor-blocking monoclonal antibody used to demonstrate Fc receptor-dependent platelet activation. DVT, deep-vein thrombosis; EIA, enzyme immunoassay; IgG, immunoglobulin G; IU, international units; IVIG, immune globulin; OD, optical density; PF4, platelet factor 4; PF4/H, platelet factor 4/heparin; PVS, polyvinyl sulfonate; VITT, vaccine-induced immune thrombotic thrombocytopenia; VTE, venous thromboembolism. 
without signs or symptoms of pulmonary embolism or cerebral venous thrombosis (CVT); however, he continues to have mild thrombocytopenia and elevated D-dimer levels despite having received a third dose of IVIG on day 72 .

\section{Case 2}

A 55-year-old male received his ChAdOx1 $\mathrm{nCoV}-19$ vaccine (day 0 ). From day 8 to day 11 , he experienced an intermittent, bitemporal headache (7/10 severity) that was pulsatile in nature, without focal neurologic deficits. On day 11, he experienced dyspnea on exertion and cough; his respiratory symptoms worsened over the next few days. On day 15 , he experienced small-volume hemoptysis (without other bleeding) and attended the emergency department. The platelet count was $103 \times 10^{9} / \mathrm{L}$; the patient's usual platelet count was $265 \times 10^{9} / \mathrm{L}$ ( $\mathbf{- \text { Fig. }}$ 1B). His D-dimer was $>20 \mathrm{mg} / \mathrm{L}$. Contrast tomography (CT) imaging of his chest demonstrated bilateral pulmonary emboli with evidence of saddle embolism. There were no echocardiographic findings of right heart strain and he was hemodynamically stable. Doppler ultrasound showed a left lower limb DVT, with partially occlusive thrombus in the distal superficial femoral vein extending into the popliteal vein. CT venography of his brain did not show CVT. He was admitted to a hospital and initiated on rivaroxaban $15 \mathrm{mg}$ twice-daily on day 16. Following the first two doses of anticoagulation, IVIG was started empirically given the clinical suspicion for VITT. He received $100 \mathrm{~g}$ IVIG $(1 \mathrm{~g} / \mathrm{kg}$ dose [weight: $100 \mathrm{~kg}$; height: $179 \mathrm{~cm}]$ ) daily for 2 days, on days 17 and 18 . The screening polyspecific PF4/polyanion EIA was positive (2.554 OD units) as were two IgG-specific EIAs (PF4/H and PF4 alone), with $>50 \%$ inhibition with high heparin; patient serum induced serotonin release in the presence of PF4 ( - Fig. 1B, inset). After two doses of IVIG, his platelet count rose quickly (peak: $488 \times 10^{9} / \mathrm{L}$ ). He was discharged home on rivaroxaban. Serial D-dimer measurements showed progressive decline and he remained clinically well (last follow up, day 138).

For both patients, repeat serological testing post-IVIG showed persistent reactivity in all three EIAs, but negative platelet activation assays (- Fig. 1A, B, insets).

\section{Discussion}

We present two patients with confirmed VITT who had mild thrombocytopenia, demonstrating that VITT can occur on a spectrum of severity. For the first patient, the onset of symptoms began 31 days post-ChAdOx $1 \mathrm{nCoV}-19$ vaccination, which is later than most other reports. ${ }^{1-5}$ The patient clinically improved with initial treatment with direct oral anticoagulant (DOAC) alone. This case illustrates that VITT should be considered-and can present clinically-more than 30 days following ChAdOx1 nCoV-19 vaccination. Current guidelines ${ }^{7,8}$ suggest considering VITT when thrombosis occurs up to day 28 postvaccination; however, delayed symptom onset occurred in our case. The second patient presented within the typically cited window postvaccination, and also responded well to DOAC with additional
IVIG. Although the ultimate outcome was favorable, he presented with pulmonary embolism, a potentially fatal thrombotic event. Despite both patients having marked elevations in D-dimer $(>20 \mathrm{mg} / \mathrm{L})$, they had only mild thrombocytopenia.

Similar to heparin-induced thrombocytopenia (HIT), VITT can present with a range of thrombocytopenia, and should still be considered even if the platelet count is above $100 \times 10^{9} /$ L. Our patients' platelet count falls were $~ 40 \%$ (patient 1 ) and $\sim 60 \%$ (patient 2 ) from their usual baseline values. Approximately 10 to $20 \%$ of patients with HIT have platelet count nadirs that are $>100 \times 10^{9} / \mathrm{L},{ }^{9}$ and VITT cases with mild thrombocytopenia have been reported. ${ }^{1,3,10} \mathrm{Al}$ though there have been several reports of patients with severe VITT, we observed two patients, presenting within a few days of one another at one hospital, both with lower limb venous thrombosis and mild thrombocytopenia, both with overall good outcomes. Although we administered high-dose IVIG to both patients, we acknowledge that favorable outcomes may have occurred even without this treatment. However, the natural history of VITT remains undefined, and given the life-threatening nature of this novel prothrombotic disorder, de-escalation of platelet and coagulation activation by high-dose IVIG is an important treatment consideration. ${ }^{11}$ High-dose IVIG has demonstrated clinical benefit ${ }^{1-4,11,12}$ in the management of VITT and is considered in current guidelines and expert consensus to be an important up-front treatment., ${ }^{7,14}$ Both patients' postIVIG serum reverted to a negative PF4-enhanced SRA despite persisting EIA reactivity (-Fig. 1A, B, insets). These data support a recent report ${ }^{11}$ indicating that the mechanism of action of IVIG is competitive inhibition of VITT antibodymediated platelet activation at the level of the platelet Fc receptors, without affecting binding of VITT antibodies to the (recently identified ${ }^{15}$ ) PF4 antigen target. Our cases thus further support the efficacy of IVIG in correcting thrombocytopenia and reducing hypercoagulability in VITT.

Author Contributions

N.G., T.A., and S.P. delivered patient care and provided clinical data; N.G. and T.A. prepared the initial manuscript draft. I.N. and D.M.A. provided laboratory supervision. M. P. supervised the clinical data collection. J.I.S. reviewed laboratory data and devised an explicatory figure. T.E.W. interpreted the data and provided critical revisions to the manuscript. All authors reviewed drafts of the paper and approved the final version of this communication.

\section{Funding}

Funding support for this work was provided by a grant from the Canadian Institutes of Health Research (CIHR \#452655) awarded to Dr. Ishac Nazy.

\section{Conflict of Interest}

T.E.W. has received lecture honoraria from Alexion and Instrumentation Laboratory, and royalties from Informa (Taylor \& Francis); has provided consulting services to Aspen Canada, Aspen Global, Bayer, CSL Behring, 
Ergomed, and Octapharma; has received research funding from Instrumentation Laboratory; and has provided expert witness testimony relating to HIT and non-HIT thrombocytopenic and coagulopathic disorders. I.N. and D.M.A. are the scientific and medical directors of the McMaster Platelet Immunology Laboratory (national HIT/VITT reference center). All other authors declare no completing interests.

\section{Acknowledgments}

The authors thank Mr. James W. Smith and Ms. Jane C. Moore for performing the platelet activation assays and enzyme immunoassays, respectively.

\section{References}

1 Greinacher A, Thiele T, Warkentin TE, Weisser K, Kyrle PA, Eichinger S. Thrombotic thrombocytopenia after ChAdOx1 nCov-19 vaccination. N Engl J Med 2021;384(22):2092-2101

2 Scully M, Singh D, Lown R, et al. Pathologic antibodies to platelet factor 4 after ChAdOx1 nCoV-19 vaccination. N Engl J Med 2021; 384(23):2202-2211

3 Schultz NH, Sørvoll IH, Michelsen AE, et al. Thrombosis and thrombocytopenia after ChAdOx1 $\mathrm{nCoV}-19$ vaccination. $\mathrm{N}$ Engl J Med 2021;384(22):2124-2130

4 Jones M, Boisvert A, Landry J, Petrasek PF. Limb ischemia and pulmonary artery thrombosis after the ChAdOx1 nCoV-19 (Oxford-AstraZeneca) vaccine: a case of vaccine-induced immune thrombotic thrombocytopenia. CMAJ 2021;193(24):E906-E910

5 Sholzberg M, Arnold DM, Laupacis A. Recognizing, managing and reporting vaccine-induced immune thrombotic thrombocytopenia. CMAJ 2021;193(24):E913-E915

6 Horsewood P, Warkentin TE, Hayward CP, Kelton JG. The epitope specificity of heparin-induced thrombocytopenia. $\mathrm{Br} J$ Haematol 1996;95(01):161-167
7 Pai M, Chan B, Stall NM, et al. Drugs \& Biologics Clinical Practice Guidelines Working Group and the Ontario COVID-19 Science Advisory Table. Vaccine induced immune thrombotic thrombocytopenia (VITT) following adenovirus vector COVID-19 vaccination. Ontario: Ontario COVID-19 Science Advisory Table; 2021. Accessed May 29, 2021 at: https://covid19-sciencetable.ca/sciencebrief/vaccine-induced-immune-thrombotic-thrombocytopeniavitt-following-adenovirus-vector-covid-19-vaccination/.

8 Nazy I, Sachs UJ, Arnold DM, et al. Recommendations for the clinical and laboratory diagnosis of VITT against COVID-19: communication from the ISTH SSC Subcommittee on Platelet Immunology. J Thromb Haemost 2021;19(06):1585-1588

9 Warkentin TE. Heparin-induced thrombocytopenia: pathogenesis and management. Br J Haematol 2003;121(04):535-555

10 Lavin M, Elder PT, O'Keeffe D, et al. Vaccine-induced immune thrombotic thrombocytopenia (VITT) - a novel clinico-pathological entity with heterogeneous clinical presentations. Br J Haematol 2021. Doi: 10.1111/bjh.17613

11 Bourguignon A, Arnold DM, Warkentin TE, et al. Adjunct immune globulin for vaccine-induced immune thrombotic thrombocytopenia. N Engl J Med 2021;385(08):720-728

12 Graf T, Thiele T, Klingebiel R, Greinacher A, Schäbitz WR, Greeve I. Immediate high-dose intravenous immunoglobulins followed by direct thrombin-inhibitor treatment is crucial for survival in SarsCovid-19-adenoviral vector vaccine-induced immune thrombotic thrombocytopenia VITT with cerebral sinus venous and portal vein thrombosis. J Neurol 2021;268(12):4483-4485

13 Pavord S, Lester W, Makris M, et al. Guidance from the Expert Haematology Panel (EHP) on Covid-19 vaccine-induced immune thrombocytopenia and thrombosis (VITT). London: British Society for Haematology, May 28, 2021

14 ISTH. ISTH interim guidance for the diagnosis and treatment of vaccine-induced immune thrombotic thrombocytopenia. Accessed July 15, 2021 at: https://cdn.ymaws.com/www.isth. org/resource/resmgr/ISTH_VITT_Guidance_2.pdf

15 Huynh A, Kelton JG, Arnold DM, Daka M, Nazy I. Antibody epitopes in vaccine-induced immune thrombotic thrombocytopaenia. Nature 2021;596(7873):565-569 